TITLE:

\title{
Time-resolved photoelectron spectroscopy of bulk liquids at ultra-low kinetic energy
}

\section{$\operatorname{AUTHOR}(\mathrm{S})$ :}

Tang, Ying; Suzuki, Yoshi-ichi; Shen, Huan; Sekiguchi, Kentaro; Kurahashi, Naoya; Nishizawa, Kiyoshi; Zuo, Peng; Suzuki, Toshinori

\section{CITATION:}

Tang, Ying ...[et al]. Time-resolved photoelectron spectrosco py of bulk liquids at ultra-low kinetic energy. Chemical Physics Letters 2010, 494(1-3): 111-116

\section{ISSUE DATE:}

2010-07-09

URL:

http://hdl.handle.net/2433/128932

\section{RIGHT:}

(C) 2010 Elsevier B.V.; This is not the published version. Please cite only the published version.; この論文は出版社版でありません。引用の際に は出版社版をご確認ご利用ください。 


\section{Time-Resolved Photoelectron Spectroscopy of Bulk}

\section{Liquids at Ultra-Low Kinetic Energy}

Ying Tang, ${ }^{1,2}$ Yoshi-ichi Suzuki, ${ }^{1,2}$ Huan Shen, ${ }^{2,4}$ Kentaro Sekiguchi, ${ }^{1,2}$ Naoya Kurahashi, ${ }^{2}$ Kiyoshi Nishizawa, ${ }^{3}$ Peng Zuo, ${ }^{3}$ and Toshinori Suzuki ${ }^{1,2,3^{*}}$

${ }^{1}$ Japan Science and Technology Agency, CREST, Sanbancho, Chiyoda-ku, Tokyo 102-0075, Japan.

${ }^{2}$ Department of Chemistry, Graduate School of Science, Kyoto University, Kyoto 606-8502, Japan.

${ }^{3}$ Chemical Dynamics Laboratory, RIKEN Advanced Science Institute, RIKEN, Wako 351-0198, Japan.

${ }^{4}$ Wuhan Institute of Physics and Mathematics, Chinese Academy of Sciences, Wuhan, People's Republic of China.

Corresponding author: suzuki@kuchem.kyoto-u.ac.jp 


\begin{abstract}
Time-resolved photoelectron spectroscopy (TR-PES) of ultrafast dynamics in solution is presented. To measure the photoelectron kinetic energy distribution (PKED) that is free from inelastic scattering in solution, photoelectrons were generated with ultra-low kinetic energies (ULKE: $<5 \mathrm{eV}$ ). Time constants of the elementary processes in the charge-transfer-to-solvent (CTTS) reaction from $\mathrm{I}^{-}$to bulk water were in excellent agreement with those obtained by transient absorption spectroscopy, demonstrating the bulk-sensitivity of TR-PES-ULKE. The analysis suggests that the CTTS reaction proceeds via two intermediates, and that $30 \%$ of the first intermediate and $70 \%$ of the second intermediate respectively are quenched by geminate recombination between the electron and the neutral iodine atom.
\end{abstract}




\section{Graphical abstract}

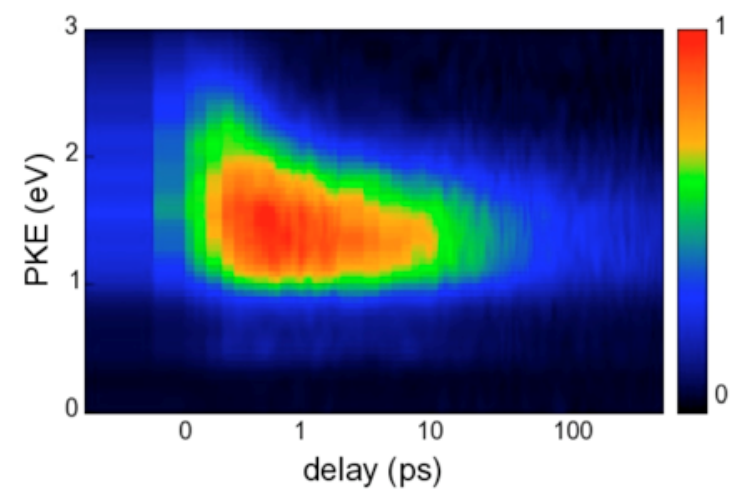

Ultrafast electronic dynamics in an aqueous solution was investigated by time-resolved photoelectron spectroscopy at ultra-low kinetic energy. 


\section{Introduction}

Time-resolved photoelectron spectroscopy (TR-PES) permits direct measurement of the ultrafast electronic dynamics in isolated molecules [1, 2], clusters [2], and surfaces [3]. However, bulk solutions have not been investigated by TR-PES, despite the electronic dynamics in solutions being critical for understanding organic, inorganic, and biochemical reactions. One technical difficulty using PES on solutions is the incompatibility of using volatile liquids with the high vacuum required for PES. However, this difficulty has been alleviated considerably by the introduction of the liquid beam (or microjet) technique $[4,5]$. TR-PES of a liquid beam was performed using extreme UV radiation $(h v=$ $38.6 \mathrm{eV})$ to study the liquid-gas phase transition in water excited by an intense IR pulse $(2.6-3.0 \mu \mathrm{m})$ [6].

A more critical problem in probing bulk liquids using PES is that electrons generated in the bulk liquid undergo both elastic and inelastic scattering with the solvent. Elastic and inelastic scattering have different effects on PES. Elastic scattering reduces only the electron flux observed within a certain solid angle outside the liquid, whereas inelastic scattering reduces both the flux and the kinetic energy (KE) of electrons; this latter effect significantly degrades the information carried by the electrons.

The inelastic mean free path (IMFP) of an electron in bulk materials has been extensively studied experimentally [7] and theoretically [8-12]. Tanuma, Powell, and Penn [8-12] have calculated the IMFPs in various elements and in inorganic and organic materials. They found that the IMFP exhibits very similar dependences on KE in different materials. The IMFP has a minimum of less than $1 \mathrm{~nm}$ in the $\mathrm{KE}$ range of $50-100 \mathrm{eV}$ and it increases monotonically on both the higher- $(>100 \mathrm{eV})$ and lowerenergy sides $(<50 \mathrm{eV})$. The extension of the IMFP at low $\mathrm{KE}$ is dramatic. As for water, Emfietzoglou et al. [13] estimated the IMFP in bulk water theoretically and obtained a value of $10 \mathrm{~nm}$ at $\mathrm{KE}=10 \mathrm{eV}$; this figure is regarded as an approximate guideline for the IMFP. The only experimental estimate of the IMFP in water is $2-4 \mathrm{~nm}$ for $\mathrm{KE}<10 \mathrm{eV}$ in amorphous ice at $14 \mathrm{~K}$ [14]. However, since amorphous 
ice contains many voids and pores that may trap electrons by $\mathrm{OH}$ dangling bonds, the IMFP in amorphous ice may differ from that in liquid water. The general energy dependence of IMFP [7-13] indicates that the largest IMFP is obtained at ultra-low $\mathrm{KE}(<5 \mathrm{eV}$ : ULKE). An electron passing through liquid water with ULKE cannot excite plasmon resonance or valence electrons in solvent water and it interact only with phonons, which makes the IMFP very long at ULKE. If the IMFP becomes longer than the probing depth (or effective attenuation length; EAL) of PES, the observed PKED will not involve inelastic-scattered photoelectrons.

At ULKE, EAL is determined by elastic scattering. EAL should also be sufficiently long to allow observation of the inside of the bulk solution. Unfortunately, neither the EAL nor the IMFP is accurately known at ULKE. Thus, PES of bulk liquids at ULKE is an unexplored field.

In this study, we report the first TR-PES of solution at ULKE. Given the uncertainty in the IMFP and the EAL at ULKE, an important objective of this study is to examine whether TR-PES-ULKE can be used to observe the bulk dynamics. To this end, we compare the time constants of elementary processes determined by TR-PES with those obtained by conventional transient absorption spectroscopy (TAS) in solution. Based on confirmation of the bulk-sensitivity of TR-PES-ULKE, we discuss the dynamics in aqueous solution elucidated by TR-PES.

Atomic halogen anions in water exhibit strong UV absorption due to charge-transfer-to-solvent (CTTS) bands [15-17]. Excited electrons in these states are quasibound and are rapidly transferred to water where they form hydrated electrons. Since CTTS is a prototypical electron transfer process in aqueous solution, its mechanism is of great interest. Mixed quantum-classical simulations predict that the CTTS reaction proceeds via an intermediate. The predicted intermediate is a contact pair consisting of a halogen atom and an electron caged in the same hydration shell ( $\mathrm{I}^{-}$case) or a solvent-separated state $\left(\mathrm{Cl}^{-}\right.$case $)$[15-17]. The weak binding energies in these complexes arise from charge-induced dipole interactions between the halogen atom and an excess electron. Excellent real-time observations 
of the CTTS reaction from $\mathrm{I}^{-}$to water have been performed by TAS in the visible to near-infrared region [17-20]. However, observation of the fastest dynamics by TAS is limited because the spectral width of the transient absorption signal at short time delays $(<400 \mathrm{fs})$ exceeds the experimental wavelength range (400-1100 nm). TR-PES observes the entire dynamics from the Franck-Condon state to the asymptotic products and provides complementary information to TAS on the CTTS dynamics.

\section{Experimental Section}

\section{(a) Apparatus}

The $0.14 \mathrm{M} \mathrm{NaI}$ solution prepared using deionized and deaerated water was introduced into a photoelectron spectrometer [21] as a laminar flow from a fused-silica-capillary nozzle with an inner diameter of $25 \mu \mathrm{m}$ and a length of $7 \mathrm{~mm}$. The liquid flow rate was $0.5 \mathrm{~mL} / \mathrm{min}$, and the Reynolds number was $\sim 530$. Previously, TAS reported that the time-profile exhibited no change over the concentration range of $0.015-0.19 \mathrm{M}$ in water [19]. Pressure in the chamber was maintained at $0.8-$ $1.0 \times 10^{-4}$ Torr by trapping the liquid beam with a liquid- $\mathrm{N}_{2}$-cooled cold finger placed downstream. A 1-kHz regeneratively amplified Ti:sapphire laser excited two optical parametric amplifiers to generate pump and probe UV pulses. These pulses were attenuated and focused onto the liquid at a point $1 \mathrm{~mm}$ downstream of the nozzle. Typical laser powers were $<70 \mathrm{~nJ}$ (pump) and $<200 \mathrm{~nJ}$ (probe). When the laser pulse energies were too high, the PKED extended toward a higher PKE. Therefore, we measured the PKED for different pulse energies first and chose sufficiently low pulse energies that do not broaden the PKED. Photoelectrons emitted from the liquid were sampled through a skimmer (5-mm $\phi)$ located $11 \mathrm{~mm}$ from the liquid and were measured with a hemispherical electron energy analyzer. Both the pump and probe laser pulses were linearly polarized. The probe laser polarization was parallel to the electron flight axis, whereas the pump laser polarization was set at the magic angle with respect to 
the axis. A strong photoelectron signal was detected only when the laser beams overlapped with the liquid beam. No photoelectron signal was observed for a pure-water beam with the pump and probe pulse energies used for aqueous NaI solution; this demonstrates that two-photon ionization of solvent water did not occur. Raman spectroscopy of a pure-water beam implies that the temperature reduction due to evaporative cooling $1 \mathrm{~mm}$ downstream from the nozzle is less than $10 \mathrm{~K}$ [22]. The energy resolution of our experiment was determined to be $40 \mathrm{meV}$ from the linewidth of the photoelectron spectrum of gaseous NO.

In photoelectron angular distribution (PAD) measurements, we used one-color two-photon ionization at $226 \mathrm{~nm}$ of $\mathrm{I}^{-}(\mathrm{aq})$ via the ${ }^{2} \mathrm{P}_{3 / 2}$ CTTS state. The linear polarization direction of the laser pulse was varied with a Berek polarization compensator (New Focus) with respect to the electron detection axis. The laser beam moved a distance of about $30 \mu \mathrm{m}$ from the target position when the polarization compensator was rotated, which was not negligible for a $25-\mu \mathrm{m}$-diameter liquid beam (the beam diameter may be slightly smaller than $25 \mu \mathrm{m}$ due to contraction after ejection from the nozzle into vacuum). Therefore, we corrected the beam pointing by monitoring the Fraunhofer diffraction of the laser beam passing through the liquid beam.

(b) Reduction of electrokinetic streaming potential and calibration of photoelectron energy

An electron ejected from a liquid is accelerated or decelerated depending on the surface charge of the liquid. A surface charge is generated when a liquid passes through a liquid beam nozzle [23, 24]. The resulting electrokinetic streaming potential $\left(\Phi_{\text {str }}\right)$ is given by

$$
\Phi_{\text {str }}=-\frac{1}{2 \pi \varepsilon_{0}} \frac{I_{\text {str }}}{v_{\text {beam }}} \ln \left(\frac{d_{\text {beam }}}{2}\right),
$$

where $I_{\text {str }}$ is the streaming current, $v_{\text {beam }}$ is the liquid beam velocity, and $d_{\text {beam }}$ is the beam diameter. In PES of liquids, the observed PKE must be corrected for $\Phi_{\text {str }}$. To minimize the calibration error and 
reduce the surface charge (which may hinder an electron from escaping into vacuum), we reduced the streaming potential by adding an electrolyte to the liquid. In the case of aqueous NaI solution discussed in the present study, the sample itself is an electrolyte; therefore, we only needed to optimize the concentration and minimize $\Phi_{\text {str }}$.

To determine the optimum concentration of NaI, we measured $\Phi_{\text {str }}$ in situ by ionizing desorbed molecules from the liquid surface. No photoelectrons were observed for gas-phase species at the pulse intensities (<200 nJ/pulse) used for TR-PES of liquids, but they were observed when the intensity was increased ( $>10 \mu \mathrm{J} /$ pulse). From a series of measurements with different salt concentrations, we found that $\Phi_{\text {str }}$ was minimized for $0.14 \mathrm{M} \mathrm{NaI}$ solution. We performed TR-PES using this optimal salt concentration.

The PKE was accurately calibrated using gaseous NO. A continuous effusive flow of NO 5\% in $\mathrm{Ar}$ was introduced into the photoionization chamber from a gas nozzle with a stagnation pressure of ca. 500 Torr, which made a partial pressure of NO to be ca. $2-5 \times 10^{-6}$ Torr in the ionization chamber. NO was ionized at the original ionization point located at $L$ ( $L$ is $11 \mathrm{~mm}$ in our apparatus) from the grounded entrance aperture of the photoelectron spectrometer by moving the liquid beam away from the ionization point by $l$ (see Fig. 1a). The liquid beam was kept flowing at the new position at $L+l$ from the aperture. The streaming potential of the liquid beam at this position generates an electric field of $\Phi_{\text {str }} /(L+l)$ along the axis of the electron flight path to the detector. The PKE observed for NO ionized at position $L$ is given by:

$$
P K E(l)=\mathrm{h} v-V B E-\frac{L}{L+l} \Phi_{s t r}+V
$$

where $V$ represents an additional (unknown) source of the PKE shift that depends on the condition of the photoelectron spectrometer. Since we know the vertical binding energy (VBE) of NO, Eq. (2) can be expressed as: 


$$
P K E(l)=P K E(N O)-\frac{L}{L+l} \Phi_{s t r}+V,
$$

where $\mathrm{PKE}(\mathrm{NO})$ is the known value for one-color two-photon ionization of $\mathrm{NO}$ at $226 \mathrm{~nm}$. By measuring the PKE shift of NO for two different positions $l_{1}$ and $l_{2}$ of the liquid beam, we obtain

$$
\left\{\begin{array}{l}
\Phi_{s t r}=\left\{\operatorname{PKE}\left(l_{1}\right)-\operatorname{PKE}\left(l_{2}\right)\right\} /\left\{\frac{L\left(l_{1}-l_{2}\right)}{\left(L+l_{1}\right)\left(L+l_{2}\right)}\right\} \\
V=\left\{\left(L+l_{1}\right) \operatorname{PKE}\left(l_{1}\right)-\left(L+l_{2}\right) \operatorname{PKE}\left(l_{2}\right)\right\} /\left(l_{1}-l_{2}\right)-\operatorname{PKE}(N O)
\end{array}\right.
$$

Thus, the VBE of the liquid is obtained from the observed PKE using:

$$
\operatorname{VBE}(\text { liq })=\mathrm{h} v-\operatorname{PKE}(\text { liq })-\Phi_{\text {str }}+V .
$$

Figure $1 \mathrm{~b}$ shows an example. In these measurements, NO was ionized using $(1+1)$ one-color twophoton ionization at $226 \mathrm{~nm}$ with a liquid beam of $0.14 \mathrm{M}$ aqueous $\mathrm{NaI}$ solution flowing near the ionization point. Locations $l=2$ and $5 \mathrm{~mm}$ from the ionization point were chosen. The results indicate that the observed PKE shifts by $25 \mathrm{meV}$ when the liquid beam is moved by $3 \mathrm{~mm}$. From this result, we obtain $\Phi_{\text {str }}=-160 \mathrm{mV}$. The PKED from NO is narrow and symmetric, indicating that there is negligible inelastic scattering of electrons in the ambient gas pressure around the liquid beam. 
(a)
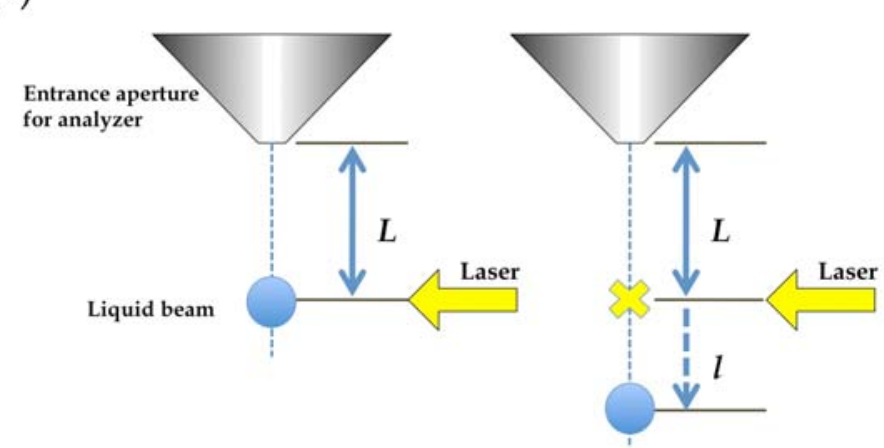

TRPES of Liquids

Calibration of PKE

(b)



Figure 1 Calibration of PKE. (a) Experimental geometry. (b) PKED observed by one-color (1+1) REMPI of $\mathrm{NO}$ at $226 \mathrm{~nm}$ with two different positions $(l=2$ or $5 \mathrm{~mm})$ of the liquid beam ( $0.14 \mathrm{M}$ aqueous NaI solution). 
(a)

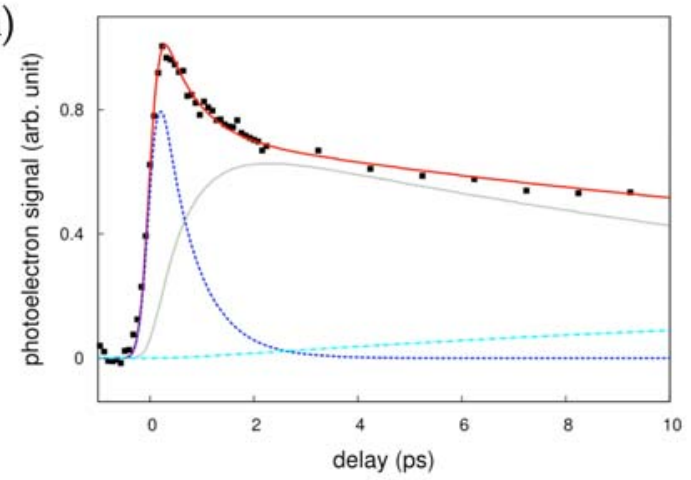

(b)

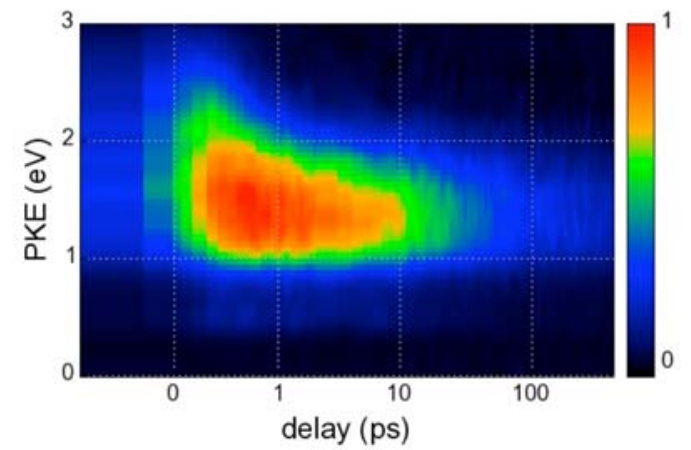

(c)

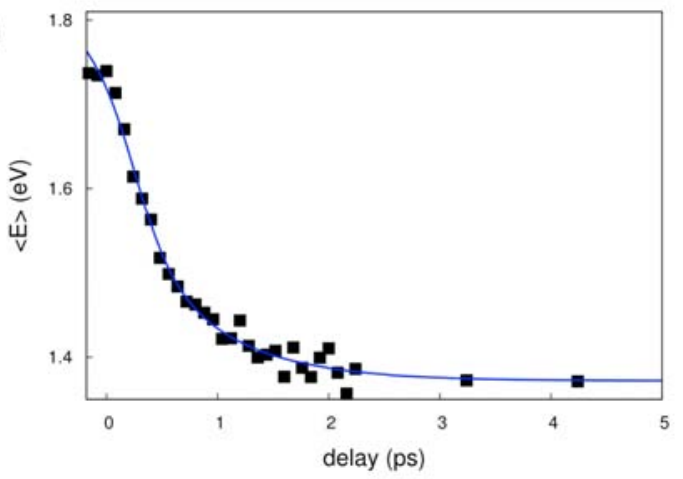

Figure 2. Results for CTTS from $\mathrm{I}^{-}$(aq) to bulk water in $0.14 \mathrm{M} \mathrm{NaI}$ aqueous solution. (a) Pump-probe time profile observed for wavelengths of $243 \mathrm{~nm}$ (pump) and $260 \mathrm{~nm}$ (probe). Black squares represent the experimental data points, and the solid red line shows the result of least-squares fitting assuming three components. The individual components are indicated in blue, light blue, and gray lines. (b) False-color plot of the PKED measured for $243 \mathrm{~nm}$ (pump) and $260 \mathrm{~nm}$ (probe). The cross-correlation of the laser pulses is $395 \mathrm{fs}$. The delay time is plotted on a logarithmic scale. A constant has been added to the actual delay $\left(t_{\text {plot }}=t_{\text {true }}+0.2\right.$ ps) to shift the entire distribution to show the data around $t=0$. The time labels and grids are presented for $t_{\text {true }}$. (c) Averaged values of PKEs at each time delay. The solid blue line shows the result of the least-squares fitting using Eq. (7) (see the text). 


\section{Results and Discussion}

(a) TR-PES of CTTS from $\mathrm{I}^{-}$to water at $\lambda_{\text {pump }}=243 \mathrm{~nm}$

We excited $\mathrm{I}^{-}$in bulk water at the red edge of the ${ }^{2} \mathrm{P}_{3 / 2}$ CTTS band $(243 \mathrm{~nm})$ and observed the subsequent dynamics by TR-PES using time-delayed 260-nm pulses. Figure 2a shows the observed pump-probe photoelectron signal intensity (an integrated area of PKED) as a function of time. The time profile indicates that multiple components are present. We performed nonlinear least-squares fitting by assuming three components and obtained time constants of $0.65,18$, and 500 ps. Iglev et al. performed TAS on the same system with 242-nm excitation and obtained three time constants of 0.2 , 0.7, and 21 ps [18]. Bradforth and coworkers have obtained similar time constants of 0.2, 0.85 and 1922 ps for $255-\mathrm{nm}$ excitation $[17,19,20]$. Notice that the smallest time constant of $0.2 \mathrm{ps}$ is absent from the TR-PES profile, indicating that the photoionization cross section and the excited state population do not change during this reaction step. The second smallest time constant, 0.7 or $0.85 \mathrm{ps}$, in TAS was determined from the spectral shift, whereas the corresponding time constant of 0.65 in TR-PES was obtained from the population decay. The time constant of 18-22 ps was determined from the population decays in both TAS and TR-PES: this time constant has been ascribed to the lifetime of an intermediate that is determined by two processes of the geminate recombination of an electron with a neutral iodine atom (time constant: $29-33 \mathrm{ps)}$ and dissociation of the intermediate to form a hydrated electron (56-70 ps) and an iodine atom [17-20].

Figure $2 \mathrm{~b}$ shows a false color map of the observed PKED for CTTS. A logarithmic scale is used for the time axis. This figure shows that PKE rapidly decreases within $1 \mathrm{ps,}$, whereas it changes very little after 1 ps. This accords with the TAS study by Iglev et al. in which they observed a rapid shift of the center photoabsorption wavelength from 1000 to $750 \mathrm{~nm}$ in the first 1 ps and no shift after 2 ps [18]. 
For quantitative analysis of the PKE shift observed by TR-PES, the averaged value of the PKED $(<E(t)>)$ was calculated from the observed time-dependent PKED, $f(E, t)$, using the following equation,

$$
\langle E(t)\rangle=\int E f(E, t) d t / \int f(E, t) d t
$$

The result is shown in Fig. 2c for the first 5 ps.

Since hydrated electrons are not produced within this ultrafast time range, the following approximation holds,

$$
\langle E(t)\rangle=\frac{E_{C T T S}\left[\mathrm{I}^{-} *\right]+E_{1}[\mathrm{I}-\mathrm{e}]+E_{2}[\mathrm{I} \bullet \mathrm{e}]}{\left[\mathrm{I}^{-} *\right]+[\mathrm{I}-\mathrm{e}]+[\mathrm{I} \bullet \mathrm{e}]}
$$

where $\left[\mathrm{I}^{-*}\right]$ represent the populations in the CTTS state, $[\mathrm{I}-\mathrm{e}]$ and $[\mathrm{I} \bullet \mathrm{e}]$ represents the first and second intermediates, respectively. $E_{i}$ is the average PKE of each species. We found that $\langle E(t)\rangle$ is expressed by three time constants. By assuming $\tau_{3}$ to be $18 \mathrm{ps}$ as determined from the time profile, least-squares fitting of $\langle E\rangle$ provided $\tau_{1}=0.22$ and $\tau_{2}=0.65$ ps. The obtained $\tau_{1}$ and $\tau_{2}$ are in reasonable agreement with those obtained by TAS [18]. Thus, both TR-PES and TAS reveal a rapid and smooth spectral shift with time constants of ca. 0.2 and $0.65-0.85$ ps. This is a remarkable agreement between the two completely different experimental methods.

(b) More detailed kinetic analysis of TR-PES

We can perform more detailed kinetics analysis by simultaneous fitting the time profile and $\langle E(t)\rangle$. However, one problem with this analysis is that the relative ionization cross sections of different chemical species are not known. When the photoelectron intensity diminishes on the elementary process from species A to B, it is not immediately clear whether the ionization cross sections of these species are similar and only a part of the population $[\mathrm{A}]$ is transformed to $[\mathrm{B}]$ or whether the population 
$[A]$ is completely transformed to $[B]$ with unit quantum yield $(\mathrm{QE})$ and the ionization cross section of $[B]$ is smaller than that of [A]. As mentioned in the previous section, the photoelectron intensity did not increase or decrease when the ${ }^{2} \mathrm{P}_{3 / 2}$ CTTS state changes to the first intermediate, despite the electronic character changing largely in this process than in later processes. Thus, it may be reasonable to assume that the ionization cross sections of all these species are the same.

Assuming identical photoionization cross sections, the kinetic equations for TR-PES of the CTTS reaction are given by:

$$
\left\{\begin{array}{l}
d[\mathrm{e}] / d t=\sigma_{i} I_{\text {probe }}(t)\left\{\left[\mathrm{I}^{-} *\right]+[\mathrm{I}-\mathrm{e}]+[\mathrm{I} \bullet e]+[\mathrm{hyd}]\right] \\
d\left[\mathrm{I}^{-} *\right] / d t=-\left(1 / \tau_{a 1}+1 / \tau_{a 2}\right)\left[\mathrm{I}^{-*} * \sigma_{a} I_{p u m p}(t)\left[\mathrm{I}^{-}\right]\right. \\
d[\mathrm{I}-\mathrm{e}] / d t=-\left(1 / \tau_{b 1}+1 / \tau_{b 2}\right)[\mathrm{I}-\mathrm{e}]+\left[\mathrm{I}^{-} *\right] / \tau_{a 1} \\
d[\mathrm{I} \bullet e] / d t=-\left(1 / \tau_{c 1}+1 / \tau_{c 2}\right)[\mathrm{I} \bullet e]+[\mathrm{I}-\mathrm{e}] / \tau_{b 1} \\
d[\mathrm{hyd}] / d t=-[\mathrm{hyd}] / \tau_{d}+[\mathrm{I} \bullet e] / \tau_{c 1}
\end{array},\right.
$$

where $[\mathrm{e}],\left[\mathrm{I}^{-}\right]$, and $[\mathrm{hyd}]$ represent the numbers of ejected photoelectrons, ground-state iodine anions, and hydrated electrons, respectively. $\sigma_{i}$ is the photoionization cross section and $I_{\text {probe }}(t)$ is the probe laser intensity, respectively. $\sigma_{a}$ is the photoabsorption cross section and $I_{\text {pump }}(t)$ is the pump laser intensity. The kinetic scheme is shown in Fig. 3. We included quenching processes characterized by $\tau_{\mathrm{b} 2}$, which was not considered in previous analyses of TAS $[18,19] . \tau_{\mathrm{a} 2}$ was included to generalize the treatment; however, we assumed it to be infinity in the present case, because no population decay was observed in this first process.

Table I summarizes the time constants obtained for each elementary process and the average PKE of each component. The ${ }^{2} \mathrm{P}_{3 / 2}$ CTTS state is completely transformed into the first intermediate. The time constant of the reaction from the CTTS state to this intermediate is calculated to be $0.17 \mathrm{ps}$, which is almost unchanged from that obtained $(0.22 \mathrm{ps})$ in the previous section. Simultaneous fitting of the time profile and the PKE shift gives an overall time constant for the population decay of the first 
intermediate of $0.8 \mathrm{ps}$, which is slightly longer than the estimate from our analysis of the time profile alone. Finally, the simulation estimates the time constants for geminate recombination of the excess electron and iodine and mutual diffusion of these species to be 28 and 59 ps, respectively. These constants are in reasonable agreement with those obtained by TAS.

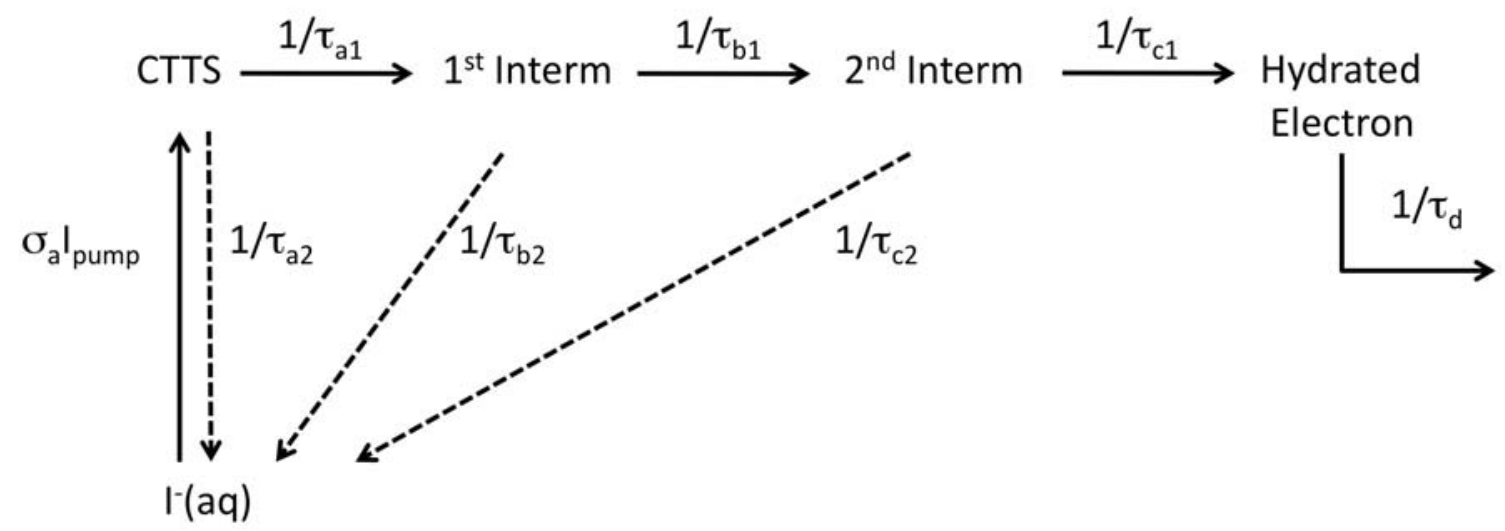

Figure $3 \quad$ Kinetic scheme used for simultaneously fitting time profile and $\langle E(t)>$

The result indicates that the overall QE of the hydrated electron is predominantly determined by the ratio of the time constants for geminate recombination $\left(\tau_{c} / \tau_{c 2} \sim 70 \%\right)$ and mutual diffusion of the excess electron and the iodine atom from the second intermediate. This has been previously elucidated by TAS [17-20]. However, the QE is also affected by quenching (or internal conversion) from the first intermediate to the ground state of $\mathrm{I}^{-}(\mathrm{aq})\left(\tau_{b} / \tau_{b 2} \sim 30 \%\right)$. Our result implies that recombination of the electron and iodine atom is an order of magnitude faster from the first intermediate than from the second intermdiate.

From the PKE observed for fully relaxed hydrated electrons for $t>>100 \mathrm{ps,} \mathrm{the} \mathrm{VBE} \mathrm{of}$ hydrated electrons in water has been estimated to be $3.27 \pm 0.1 \mathrm{eV}[25]$. The VBEs of the CTTS state, the first intermediate, and the second intermediate are estimated to be smaller than that of hydrated electrons by ca. 600, 200, and $20 \mathrm{meV}$, respectively. Previously, the well depth of the second 
intermediate has been estimated to be $\sim 3 \mathrm{k}_{\mathrm{B}} T(80 \mathrm{meV})$ with respect to the asymptotic product pair of a hydrated electron and an iodine atom. If the interaction between an iodine atom and a void (a vacancy in bulk water network after the excess electron has left) is weak, VBE should diminish when the second intermediate dissociates into a hydrated electron and a neutral iodine atom. On the contrary, the observed VBE slightly increases, indicating that the attractive interaction between an iodine atom and a void similar to or even stronger than that between an iodine atom and a hydrated electron.

The structures of the first and second reaction intermediates cannot be determined from the limited information available to us. Therefore, we mention only some important points here. Let us consider the reverse reaction from a free hydrated electron and a hydtrated iodine atom to the second intermediate. The electron and the atom respectively have hydrophilic and hydrophobic interactions with water. The electron and the atom approach each other and form a solvent-separated state. If there is a high-energy barrier for the two hydration shells to merge, the solvent-separated state will be the second intermediate. On the other hand, if there is negligible barrier, the contact pair of an electron and an iodine atom caged in the same hydration shell will be the second intermediate. Previously, a solventseparated state and a contact pair have been suggested as the intermediates in CTTS from $\mathrm{Cl}^{-}$to water and $\mathrm{I}^{-}$to water, respectively $[15,16]$.

The dynamics from the CTTS state to the first intermediate is associated with the reduction of VBE as large as $0.6 \mathrm{eV}$. This process is expected to involve rearrangement of a hydration shell to accommodate a diffuse electron cloud in the CTTS state and the detachment of the electron from $\mathrm{I}^{-}$. It is possible that these two occur on a single adiabatic potential energy surface. TR-PES suggests that the first intermediate has an order of magnitude larger rate of geminate recombination than the second reaction intermediate. We speculate that this is because the first intermediate is internally hotter than the second one and/or the electron iodine distance is closer in the first intermediate than the second one. The reduction of $\mathrm{VBE}$ in the second step from the first to second intermediate is $0.2 \mathrm{eV}$ : this is presumably 
a solvation dynamics with or without the change from a contact pair configuration to a solventseparated configuration.

Time constants (ps) and average PKE (eV) obtained by simultaneous fitting of

Table I observed time profile and $\langle\mathrm{E}(\mathrm{t})\rangle$

\begin{tabular}{|c|c|c|c|c|c|}
\hline$\tau_{\mathrm{a}}$ & $0.17(5)^{a}$ & $\tau_{\mathrm{a} 1}$ & $0.17(5)$ & $\tau_{\mathrm{a} 2}$ & $\infty$ \\
\hline$\tau_{\mathrm{b}}$ & $0.8(2)^{a}$ & $\tau_{\mathrm{b} 1}$ & $1.3(1)$ & $\tau_{\mathrm{b} 2}$ & $2.9(5)$ \\
\hline$\tau_{\mathrm{c}}$ & $19(3)^{a}$ & $\tau_{\mathrm{c} 1}$ & $59(20)$ & $\tau_{\mathrm{c} 2}$ & $28(6)$ \\
\hline$\tau_{d}$ & $490^{b}$ & & & & \\
\hline $\mathrm{E}_{\mathrm{CTTS}}$ & $2.14(10)$ & & & & \\
\hline$E_{1}$ & 1.71(10) & & & & \\
\hline$E_{2}$ & $1.54(10)$ & & & & \\
\hline $\mathrm{E}_{\mathrm{HE}}$ & $1.52(10)$ & & & & \\
\hline
\end{tabular}

(c) Effects of salt concentration and liquid temperature

For the following reasons, salt and temperature are not considered to affect the PKE and the VBE. In the dielectric continuum model, the VBE of a hydrated electron in bulk water is expressed by [26]

$$
V D E=\left(1+\frac{1}{\varepsilon_{o p}}-\frac{2}{\varepsilon_{s}}\right) \frac{e^{2}}{2 a}
$$

where $a$ is the cavity radius, and $\varepsilon_{\mathrm{op}}$ and $\varepsilon_{\mathrm{s}}$ are respectively the optical and static dielectric constants of water. For pure water, $\varepsilon_{\mathrm{op}}$ is 1.8 and $\varepsilon_{\mathrm{s}}$ is 80 at $293 \mathrm{~K}$ [27]. The effect of salt on the VBE is estimated using Eq. (10) from the dielectric constant of a sample solution. The dielectric constant for 0.148 M $\mathrm{NaI}$ solution has been reported to be $\varepsilon_{\mathrm{s}}=76.6$ at $298 \mathrm{~K}$ [28]. Assuming that $\varepsilon_{\mathrm{op}}$ is not affected by salt, we find that the VBE in $0.148 \mathrm{M} \mathrm{NaI}$ solution is only $0.1 \%$ smaller than that in pure water. This difference corresponds to less than $10 \mathrm{meV}$, which is negligible compared with our experimental error. 
When the water temperature decreases, $\varepsilon_{\mathrm{s}}$ increases. At $283 \mathrm{~K}, \varepsilon_{\mathrm{s}}$ is 84 for pure water [29], which shifts the VBE by less than $0.1 \%$. Thus, salt and temperature are found not to affect the PKE and the VBE.

\section{(d) PAD measurements}

We observed the photoelectron intensity for different polarization directions of the laser light (see Fig. 4). For this measurement, we used one-color two-photon ionization of aqueous NaI solution at 226 $\mathrm{nm}$ and a single laser pulse functioned as both the pump and probe (i.e., there was zero time delay). Here, an angle of $0^{\circ}$ indicates that the laser polarization is parallel to the electron detection axis. The result shows that the polarization dependence is small. For a more quantitative discussion, we may express the PAD as:

$$
I(\theta) \approx 1+\beta_{2} P_{2}(\cos \theta)
$$

where $\theta$ is the angle between the electron detection axis and laser polarization, and $P_{2}$ is the secondorder Legendre polynomial. We neglected the fourth-order term $P_{4}$. Our analysis, which considers the $14^{\circ}$ apex solid angle of our electron detection system, indicates that $\beta_{2}$ is less than 0.1 . The intrinsic photoelectron angular anisotropy in photodetachment of $\mathrm{I}^{-}$in bulk water is not known. If the original PAD from $\mathrm{I}^{-}$in water is highly anisotropic, the present result implies that extensive elastic scattering of electrons occurs in solution to wash out the angular anisotropy. However, since this original PAD is not known, we conclude only that the present result is consistent with the presence of elastic scattering of electrons in solution. 


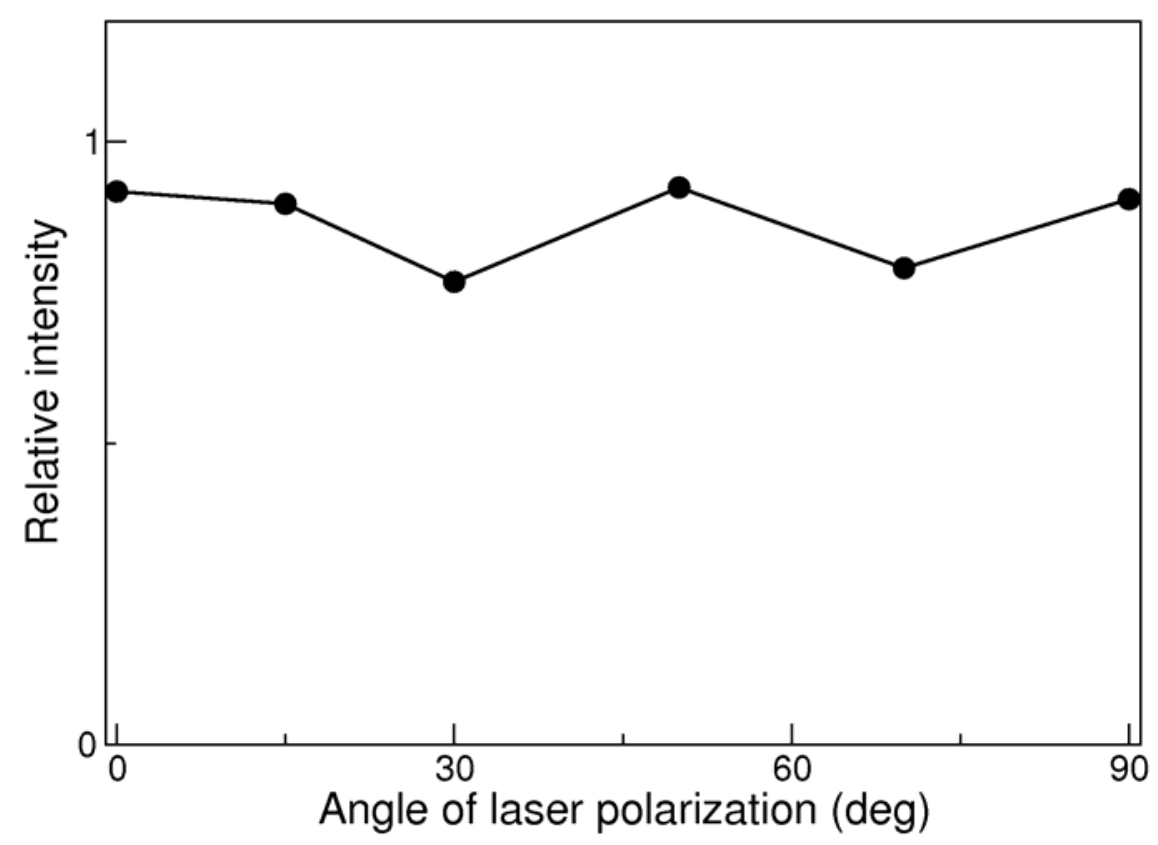

Figure 4 Photoelectron intensity as a function of laser polarization direction. One-color twophoton ionization of $\mathrm{I}^{-}(\mathrm{aq}) \mathrm{via}^{2} \mathrm{P}_{3 / 2}$ CTTS state at $226 \mathrm{~nm}$ was employed.

\section{Conclusion}

This Letter presented TR-PES-ULKE experiment and detailed analysis of the time profile of the photoelectron signal intensity and the spectral shift. We also described the procedures to measure the streaming potential of a liquid beam and calibrate the observed PKE using multi-photon ionization of NO. The time constants determined by TR-PES-ULKE are in notable agreement with previous results obtained by TAS, establishing firmly the bulk-sensitivity of TR-PES-ULKE. It is important to establish the bulk-sensitivity, since this study is the first attempt to observe a bulk solution with TR-PES-ULKE. The results obtained for the CTTS reaction from $\mathrm{I}^{-}$to water suggest that the CTTS excited state is adiabatically transformed into the first intermediate, whereas the geminate recombination quenches both of the first and the second intermediates substantially. The VBE determined for the hydrated electrons is unambiguously assigned to the value for the internal state and not the surface state. The PAD was found to be isotropic, which is consistent with elastic scattering anticipated in solution. The 
present study demonstrated that TR-PES-ULKE is highly useful for investigating the electron dynamics in bulk solutions.

\section{Note added in proof}

Very recently, TR-PES-ULKE was applied to solvated electrons in methanol and ethanol, and their vertical electron binding energies were determined [30].

Acknowledgment TS thanks Drs. S. Tanuma, A. P. Pathak, and I. Plante for kindly suggesting references about the IMFP. We dedicate this work to the late Professor Tamotsu Kondow who pioneered in laser ionization mass spectrometry using liquid beams.

(1) T. Suzuki, Annu. Rev. Phys. Chem. 57 (2006) 555.

(2) I. V. Hertel, W. Radloff, Rep. Prog. Phys. 69 (2006) 1897.

(3) J. Zhao, B. Li, K. Onda, M. Feng, H. Petek, Chem. Rev. 106 (2006) 4402.

(4) H. Siegbahn, K. Siegbahn, J. Electron Spectrosc. Relat. Phenom. 2 (1973) 319.

(5) M. Faubel, in Photoionization and Photodetachment Part II, $10 B$ of Advanced Series in Physical Chemistry, World Scientific, Singapore (1999).

(6) O. Link, E. Lugovoy, K. Siefermann, Y. Liu, M. Faubel, B. Abel, Appl. Phys. A 96 (2009) 117.

(7) M. P. Seah, W. A. Dench, Surf. Interface Anal. 1 (1979) 2.

(8) S. Tanuma, C. J. Powell, D. R. Penn, Surf. Interface Anal. 11 (1988) 577.

(9) S. Tanuma, C. J. Powell, D. R. Penn, Surf. Interface Anal. 17 (1991) 911. 
(10) S. Tanuma, C. J. Powell, D. R. Penn, Surf. Interface Anal. 17 (1991) 922.

(11) S. Tanuma, C. J. Powell, D. R. Penn, Surf. Interface Anal. 20 (1993) 77.

(12) S. Tanuma, C. J. Powell, D. R. Penn, Surf. Interface Anal. 21 (1993) 165.

(13) D. Emfietzoglou, I. Kyriakou, I. Abril, R. Garcia-Molina, I. D. Petsalakis, H. Nikjoo, A. Pathak, Nucl. Instrum. Meth. Phys. Res. B 267 (2009) 45.

(14) M. Michaud, A. Wen, L. Sanche Radiat. Res. 159 (2003) 3.

(15) W.-S. Sheu, P. J. Rossky, J. Am. Chem. Soc. 115 (1993) 7729.

(16) A. Staib, D. Borgis, J. Chem. Phys. 104 (1996) 9027.

(17) X. Chen, S. E. Bradforth, Annu. Rev. Phys. Chem. 59 (2008) 203.

(18) H. Iglev, A. Trifonov, A. Thaller, I. Buchvarov, T. Fiebig, A. Laubereau, Chem. Phys. Lett. 403 (2005) 198.

(19) J. A. Kloepfer, V. H. Vilchiz, V. A. Lenchenkov, A. C. Germaine, S. E. Bradforth, J. Chem. Phys. 113 (2000) 6288.

(20) V. H. Vilchiz, X. C. Chen, J. A. Kloepfer, S. E. Bradforth, Radiat. Phys. Chem. 72 (2005) 159.

(21) M. Oku et al., J. Phys. Chem. A 112 (2008) 2293.

(22) C. D. Cappa, W. S. Drisdell, J. D. Smith, R. J. Saykally, R. C. Cohen, J. Phys. Chem. B 109 (2005) 24391.

(23) M. Faubel, B. Steiner, J. P. Toennies, J. Chem. Phys. 106 (1997) 9013. 
(24) W. L. Holstein, L. J. Hayes, E. M. C. Robinson, G. S. Laurence, M. A. Buntine, J. Phys. Chem. B 103 (1999) 3035.

(25) Y. Tang, H. Shen, K. Sekiguchi, N. Kurahashi, T. Mizuno, Y. Suzuki, T. Suzuki, Phys. Chem. Chem. Phys. 12 (2010) 3653.

(26) M. A. Aguilar, F. J. Olivares del Valle, J. Tomasi, J. Chem. Phys. 98 (1993) 7375.

(27) J. H. Christensen, A. J. Smith, R. B. Reed, K. L. Elmore, J. Chem. Eng. Data 11 (1966) 60.

(28) W. Wachter, W. Kunz, R. Buchner, G. Hefter, J. Phys. Chem. A 109 (2005) 8675.

(29) H. Kienitz, K. N. Marsh, Pure Appl. Chem. 53 (1981) 1847.

(30) H. Shen, N. Kurahashi, T. Horio, K. Sekiguchi, T. Suzuki, Chem. Lett. 39 (2010) 668. 